\title{
Fungal endophthalmitis in an elderly woman: differing responses in each eye
}

This article was published in the following Dove Press journal:

International Medical Case Reports Journal

8 June 2017

Number of times this article has been viewed

\section{Rei Sakata ${ }^{1,2}$ \\ Jiro Numaga'}

'Department of Ophthalmology, Tokyo Metropolitan Geriatric Hospital, ${ }^{2}$ Department of Ophthalmology, Graduate School of Medicine, University of Tokyo, Tokyo, Japan
Correspondence: Rei Sakata

Department of Ophthalmology, Graduate School of Medicine, University of Tokyo, 7-3-I Hongo Bunkyo-ku,

Tokyo II 3-8655, Japan

$\mathrm{Tel}+8 \mathrm{I} 338 \mathrm{I} 554 \mathrm{II}$

Fax $+8 I 358008728$

Email reisakata-tky@umin.ac.jp
Abstract: Candida endophthalmitis is caused by hematogenous metastasis of the yeast. To ensure early diagnosis, serodiagnosis, or surveillance culturing is usually performed. If fungemia is apparent upon successive serological testing (in at least two tests: blood culture and/or measurement of $\beta$-D-glucan level), ophthalmic consultation is necessary, even if no ophthalmic complaint is evident. This is because early detection with prompt prescription of systemic antifungal agents inhibits progression of the distinctive cholioretinitis. If the initial fungemia is not promptly diagnosed, or if it is missed, fungal endophthalmitis may develop, associated with a poor prognosis in terms of visual acuity. Here, we report on a case of Candida endophthalmitis in a 92-year-old woman with severe visual disturbance in one eye that was first diagnosed on ophthalmic examination. It was already difficult to recover. Fungemic systemic/ ophthalmic symptoms must be checked in the early stage; in addition, prompt ophthalmological consultation is essential.

Keywords: Candida endophthalmitis, intravenous hyperalimentation, ophthalmic examination

\section{Introduction}

Endogenous fungal endophthalmitis, especially caused by Candida, is usually seen in patients under intravenous hyperalimentation (IVH) or with indwelling lines. Apart from catheter-related factors, supposed risk factors include abdominal operations, major cardiopulmonary vascular surgery, diabetes mellitus, other compromising conditions, immunocompromised status, and neutropenia. ${ }^{1,2}$ These significant conditions continue to be of concern to medical personnel, including ophthalmologists. Some patients continue to experience poor visual prognoses despite the prescription of systemic antifungal drugs. ${ }^{3}$ However, early detection and treatment generally allow the progression of the distinctive cholioretinitis to be controlled.

Usually, fungal endophthalmitis is identified via systemic examination (a blood culture positive for a fungus [i.e., fungemia] in those with prolonged fever). A recent report indicates that the frequency of fungal endophthalmitis among patients with fungemia is relatively low; it was speculated that anti-fungal treatments had already been administered prior to ophthalmic examination. ${ }^{4}$

In the early stage of the disease, a chorioretinal lesion is evident upon fundus examination. Next, a small circle of yellowish-white spots (an exudate) appear on the retina. If the anterior chamber becomes inflamed, this may trigger the development of anterior synechiae, iris rubeosis, and (secondary) glaucoma. 
Here, we report a case of Candida fungemia in a 92-yearold woman, first revealed by ophthalmic examination (i.e., before any systemic survey was performed). On her first outpatient visit, one eye was already seriously inflamed and the visual acuity of that eye had already deteriorated to 0.02 , whereas almost no inflammation was evident in the other eye (visual acuity, 0.4).

\section{Case report}

A 92-year-old woman, with no history of a systemic or ophthalmic disorder, no history of any prior operation, and no dementia, underwent cardiovascular surgery (placement of a total, aortic body, blood vessel prosthesis, with an open stent graft) to treat a thoracic aortic aneurysm (July 30, 2015). The surgery was successful, and she was maintained on IVH for 1 month. After IVH decannulation, she recovered well and did not complain about her eyes.

At 1 month after decannulation, she developed conjunctival hyperemia and pain in her left eye. A laboratory workup revealed a white blood cell count of $8,830 / \mu \mathrm{L}$ and a C-reactive protein (CRP) level of $1.32 \mathrm{mg} / \mathrm{dL}$. On her initial visit to our Department of Ophthalmology, her visual acuity was 0.4 in the right eye and 0.02 in the left eye. The left eye exhibited severe inflammation of the anterior chamber (cells $3+$, flares 3+ with many fine keratic precipitates; Figure 1). Fundus examination was impossible because the entire anterior chamber was covered with synechiae. Conversely, in the right eye, a cataract that reduced the patient's visual acuity somewhat (to 0.4) was evident, but no inflammation was apparent in the anterior segment or the retinal vitreous body. We scheduled a blood evaluation and culture and a culture of fluid from the anterior chamber of the left eye. The condition continued to deteriorate rapidly; iris rubeosis and hypopyon newly emerged in the left eye. Fundus examination remained impossible. We used B-mode echography to confirm that the retina was not detached and that high-intensity opacities of the vitreous body (opacitas corporis vitrei [OCV]) were absent (Figure 2). The right eye had also worsened; new find-

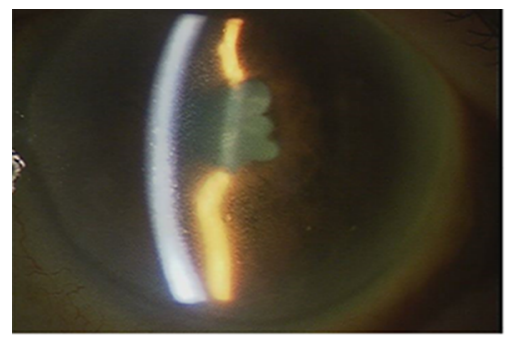

Figure I Anterior segment photograph of the left eye. Note: Severe inflammation of the anterior chamber and entire anterior synechiae.

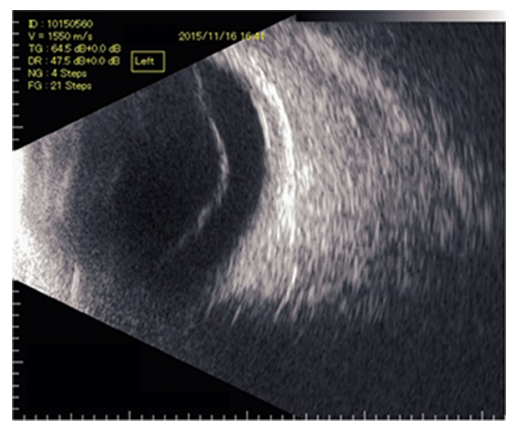

Figure 2 B-scan ultrasonography of the left eye.

Note: The retina was thought to be not detached, and high-intensity opacities of the vitreous body were also absent.

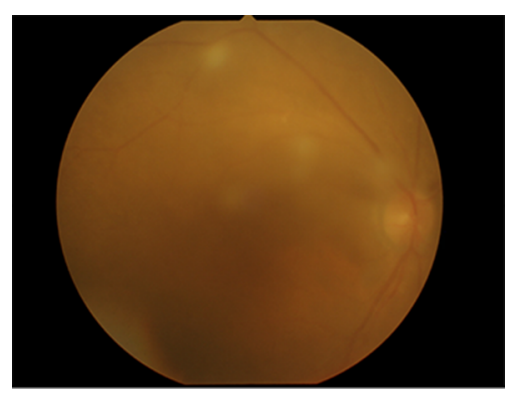

Figure 3 String of pearls OCV in right eye.

Note: OCV was seen continuously from the optic disc.

Abbreviation: OCV, opacitas corporis vitrei.

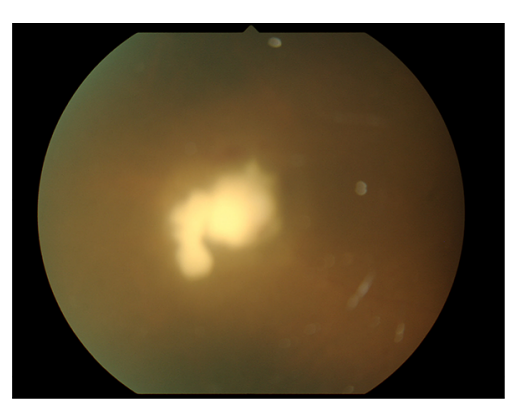

Figure 4 Fungal mass on the right retina.

Notes: A xanthochromistic fungal mass developed on the right temporal retina during systemic therapy. The photo was hazy due to OCV.

Abbreviation: OCV, opacitas corporis vitrei.

ings included beaded OCV (Figure 3), a granular exudate, vascular occlusion or angiogenesis, and a xanthochromistic fungal mass (Figure 4). Neither retinal exudation nor fundal hemorrhage was evident.

The blood results were as follows: leukocytes, $6,040 / \mu \mathrm{L}$, and CRP, $1.93 \mathrm{mg} / \mathrm{dL}$. Neither Aspergillus nor Candida antigen was detected, but the $\beta$-D-glucan level was slightly elevated, at $22 \mathrm{pg} / \mathrm{mL}$ (normal $<20 \mathrm{pg} / \mathrm{mL}$ ). Candida was detected after $45 \mathrm{~h}$ of blood culture (there was no susceptibility test result at this time); however, no microbe was cultured from the aqueous humor of the anterior chamber. We had 
already commenced empirical systemic anti-fungal therapy (fluconazole). However, none of the slight inflammation, fungal mass, or beaded OCV of the right eye shrank or disappeared. In the left eye, visual acuity deteriorated to the point of light perception; the major inflammation did not improve. A fluconazole-resistant fungal infection was of course considered; we thus planned to change the drug. However, the patient's general condition worsened rapidly because of an infection with methicillin-resistant Staphylococcus aureus. Despite best efforts to stabilize her whole-body condition, she passed away within 1 month. A detailed ophthalmological examination and invasive ophthalmic treatment were thus not possible at the outpatient clinic over the last month.

The patient's family has provided written informed consent for the publication of the case report.

\section{Discussion}

It is widely known that the risk of endogenous fungal endophthalmitis is high in patients undergoing IVH and those with intravenous indwelling catheters. Early diagnosis and treatment are essential. When a fungal infection is suspected, it is often possible to prove the suspicion by performing a rapid test prior to ophthalmic examination. Antifungal drugs commonly cure typical endophthalmitis. Thus, progression to blindness is rare. Although serum tests allowing the early diagnosis of candidemia are under development, such tests may be negative in some patients with fungal eye infections.

Up to $13 \%$ of all cases of ocular candidiasis may be missed upon initial evaluation. ${ }^{1}$ Thus, if a patient does not exhibit any characteristic risk factor and superficially appears to be in good health, it can be difficult to distinguish ocular candidiasis from uveitis. Some cases exhibit no typical findings on fundus examination; thus, fungal endophthalmitis cannot be confirmed. It is then necessary to comprehensively examine the clinical findings in terms of the number of inflammatory cells in the anterior chamber, the extent of vitreous opacity, and other clinical data. If a fungus is cultured from blood, fundus examination is essential. If fungemia persists even when the fundus is not abnormal, periodic fundus evaluation is nonetheless required.

In this case, at the first ophthalmology visit, only one eye exhibited strong inflammation with synechiae in the iris. Further, as 1 month had passed since IVH removal and as blood tests had revealed minimal inflammation, we initially considered that fungal endophthalmitis was unlikely. This is because the average time from the onset of subclinical fungemia to an ophthalmology consultation is usually short. ${ }^{4}$ As differential diagnoses, we considered intrinsic bacterial endophthalmitis, miliary tuberculosis, and diabetic retinopathy. Intrinsic bacterial endophthalmitis was ruled out because the clinical course is usually rapid. The other two possibilities were also eliminated by reference to the clinical symptoms and history. It was important to check that there was no history of (especially) persistent fever during the time of IVH.

When a catheter-related bloodstream infection (CRBSI) is suspected, a catheter culture should be performed; however, if there is no evidence of infection (e.g., fever), routine culture of the catheter tip is not always necessary. ${ }^{5}$ This is because clinical symptoms afford only poor sensitivity and specificity in the context of a CRBSI diagnosis. Even pyrexia, which affords the highest sensitivity, is not adequately sensitive.

In a hospital with no resident ophthalmologist, endophthalmitis must be diagnosed from symptoms other than those of the eyes. Normally, patients with fungal endophthalmitis have few subjective symptoms (vision may be slightly compromised in one eye). Patients who are distressed or not fully conscious may be unable to voice their complaints. Diagnosis and early treatment are likely to be delayed in such cases. Thus, it is necessary to continuously and carefully monitor the eye condition (in terms of floaters, decreased vision, hyperemia, and eye pain) and the laboratory findings (white blood cell count, CRP level, and erythrocyte sedimentation rate) of patients with suspected fungal endophthalmitis. This is because fulminant cases (with bacterial endophthalmitis) are rare.

Candida is the most common causative fungus, and azole antifungal drugs are particularly effective. Mild cases may be cured with systemic drugs; however, some drugs are in common use, reducing fungal susceptibility and patient tolerance. Thus, the condition is becoming increasingly difficult to treat. It is important to evaluate drug susceptibility to allow the selection of an appropriate antifungal. The dosage period usually ranges from 3 weeks to 3 months, and the dose is, gradually, decreased as the condition of the fundus improves. If no improvement is evident upon treatment, a change in drug should be considered. This time, we started empirical treatment before blood culture results were available. After the first culture test was positive, no fungus was detected from the blood culture.

Furthermore, if the symptoms continue to worsen despite systemic antifungal administration, vitreous surgery should be considered after evaluating the patient's systemic condition. Vitreous surgery has certain advantages, including 1) the fungus is removed from the eye; 2) the drug transfer effect is increased; 3) posterior vitreous detachment is avoided, 
as is unwanted cellular proliferation; and 4) confirmatory diagnostic and drug susceptibility tests can be performed. These allow anti-fungals to be chosen using the principles of evidence-based medicine; also, the drugs can be directly instilled into the eye. In this case, vitreous surgery was not our choice of treatment because the patient was elderly (92 years of age) and bedridden. Also, direct treatments, such as vitreous tap or injection, were difficult, given her poor physical condition. The use of vitreous therapies in older patients requires consideration of the expected lifespan, any systemic conditions, and the condition of the eye. Standardized surgical models may not adequately address the needs of the very elderly.

Fungemia progression is regularly slow; early detection and treatment can prevent retinitis. However, as in this case, subclinical fungemia progresses slowly over time in the absence of ocular symptoms. The lessons are, first, that the symptoms of endophthalmitis might be severe even on the first ophthalmological examination and, in such a case, the prognosis might be poor. Second, the fungemic systemic/ ophthalmic symptoms in the very elderly might differ from those in others, making an early ophthalmological consultation essential. A judgment on when and why anti-fungal agents should begin depends on both the patient's systemic and, principally, ophthalmic features.

\section{Disclosure}

The authors report no conflicts of interest in this work.

\section{References}

1. Krishna R, Amuh D, Lowder CY, Gordon SM, Adal KA, Hall G. Should all patients with candidaemia have an ophthalmic examination to rule out ocular candidiasis? Eye (Lond). 2000;14(pt 1):30-34.

2. Adam MK, Vahedi S, Nichols MM, et al. Inpatient ophthalmology consultation for fungemia: prevalence of ocular involvement and necessity of funduscopic screening. Am J Ophthalmol. 2015;160(5): 1078. e2-1083.e2.

3. Tanaka H, Ishida K, Yamada W, Nishida T, Mochizuki K, Kawakami H. Study of ocular candidiasis during nine-year period. J Infect Chemother. 2016;22(3):149-156.

4. Dozier CC, Tarantola RM, Jiramongkolchai K, Donahue SP. Fungal eye disease at a tertiary care center: the utility of routine inpatient consultation. Ophthalmology. 2011;118(8):1671-1676.

5. Mermel LA, Allon M, Bouza E, et al. Clinical practice guidelines for the diagnosis and management of intravascular catheter-related infection: 2009 Update by the Infectious Diseases Society of America. Clin Infect Dis. 2009;49(1):1-45.
International Medical Case Reports Journal

\section{Publish your work in this journal}

The International Medical Case Reports Journal is an international, peer-reviewed open-access journal publishing original case reports from all medical specialties. Previously unpublished medical posters are also accepted relating to any area of clinical or preclinical science. Submissions should not normally exceed 2,000 words or

\section{Dovepress}

4 published pages including figures, diagrams and references. The manuscript management system is completely online and includes a very quick and fair peer-review system, which is all easy to use. Visit $\mathrm{http}: / /$ www.dovepress.com/testimonials.php to read real quotes from published authors. 